\title{
Pigments precipitated from chromate post-galvanic solutions in emulsion systems
}

\author{
Beata Klapiszewska, Andrzej Krysztafkiewicz, Teofil Jesionowski \\ Poznan University of Technology, Institute of Chemical Technology and Engineering, pl. M. Sklodowskiej-Curie 2, \\ 60-965 Poznań, Poland, e-mail: teofil.jesionowski@put.poznan.pl
}

\begin{abstract}
Studies were conducted on the production of chromium(III) silicates, the green pro-ecological pigments. The pigments were precipitated from sodium silicate and chromium(III) sulphate solutions in the system of two emulsions prepared in hexane in the presence of a non-ionic surfactant as an emulsifier. The chromium(III) sulphate represented a reduction product of chromate(VI) compounds present in post-galvanic wastes. The reduction agent involved metanal in an acidic medium. The obtained products were subjected to a comprehensive physicochemical analysis, their dispersive and morphological properties were determined.

The precipitated green products exhibiting intense colour, were uniform and their particles manifested a low tendency to form agglomerate structures.
\end{abstract}

Keywords: green pigments, precipitated chromium(III) silicates, emulsion system, post-galvanic wastes.

Presented at VII Conference Wasteless Technologies and Waste Management in Chemical Industry and Agriculture, Międzyzdroje, 12 - 15 June, 2007.

\section{INTRODUCTION}

Highly dispersed silicates (of calcium, magnesium, aluminium, zinc, etc.) find application as insoluble components of rubber mixtures and in most plastics $^{1-3}$. They are termed the fillers. The advantage of using silicate fillers involves not only the improved physico-mechanical properties of the filled composites but also the fact that silicates belong to the so called lucid fillers, i.e. they do not alter the colour of the materials. Synthetic silicates find also application as inorganic pigments and fillers of paints and varnishes ${ }^{4-6}$.

Synthetic inorganic pigments used to be obtained by a chemical synthesis involving the precipitation of sediments. For the purpose coloured salts or oxides of non-ferrous metals are used ${ }^{7}$. For the precipitation of highly dispersed pigments various waste compounds, which contain nonferrous metal cations, e.g. post-galvanic wastes originating from processes of chromium or chromate coating, can successfully be used ${ }^{8}$.

\section{EXPERIMENTAL}

In the studies post-galvanic wastes originating from chromate coating were used. At first, chromates(VI) in the waste solutions were reduced using metanal in a medium of sulphuric acid.

$\mathrm{K}_{2} \mathrm{Cr}_{2} \mathrm{O}_{7}+3 \mathrm{HCHO}+4 \mathrm{H}_{2} \mathrm{SO}_{4} \rightarrow \mathrm{K}_{2} \mathrm{SO}_{4}+\mathrm{Cr}_{2}\left(\mathrm{SO}_{4}\right)_{3}$ $+3 \mathrm{HCOOH}+4 \mathrm{H}_{2} \mathrm{O}$

The reaction secured a complete reduction of $\mathrm{Cr}^{+6}$ to $\mathrm{Cr}^{+3}$. Following the reduction, the content of $\mathrm{Cr}^{+6}$ was checked in the reaction system.

Subsequently, the reduced solution containing chromium(III) salts was used to precipitate the green pigment, chromium(III) silicate. The precipitation was performed in an emulsion system, at various temperatures. The organic phase of the emulsions (E1 and E2) involved cyclohexane and a non-ionic surfactant, Rokanol K7 (PCC Rokita SA) was used as an emulsifier. The silicate was precipitated dosing emulsions E1 and E2. In emulsion E1 5\% sodium silicate solution was applied,

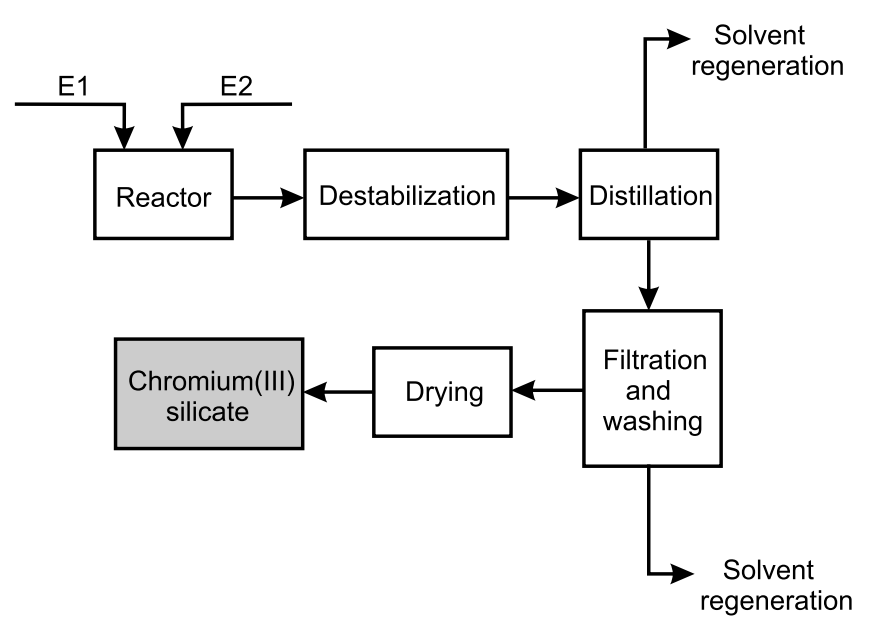

Figure 1. Schematic diagram of chromium(III) silicate precipitation in a system of two emulsions

while in emulsion E2 5\% solution of chromium(III) sulphate was used.

The procedure of obtaining in this way chromium(III) silicates is outlined in Fig.1.

The precipitated silicate pigments were subjected to analysis of principal physicochemical parameters, including particle size, the morphology of the pigment particle surface and particle size distribution. The latter was determined by dynamic light scattering using ZetaPlus apparatus (Brookhaven Instruments Co.). Surface morphology of pigment particles was defined using scanning electron microscopy (SEM) in the Philips SEM 515 instrument. The green colour intensity of the obtained chromium(III) silicates was determined in a spectrocolorimeter Specbos 4000 (JETI Technische Instrumente $\mathrm{GmbH}$ ) using the colour space system CIE $\mathrm{L}^{*} \mathrm{a}^{*} \mathrm{~b}^{*}$.

The studies permitted to determine all the indispensable variables documenting the quality of the obtained products. 
Table 1. Principal physicochemical properties of chromium(III) silicates obtained by the precipitation in the system of two emulsions at various temperatures

\begin{tabular}{|c|c|c|c|c|c|}
\hline $\begin{array}{c}\text { Sample } \\
\text { No. }\end{array}$ & $\begin{array}{c}\text { Temp., } \\
{ }^{\circ} \mathrm{C}\end{array}$ & $\begin{array}{c}\text { Bulk } \\
\text { density, } \\
\mathrm{g} / \mathrm{dm}^{3}\end{array}$ & $\begin{array}{c}\text { Capacity to absorb water, } \\
\mathrm{cm}^{3} / 100 \mathrm{~g}\end{array}$ & $\begin{array}{c}\text { Capacity to absorb dibutyl phthalate, } \\
\mathrm{cm}^{3} / 100 \mathrm{~g}\end{array}$ & $\begin{array}{c}\text { Capacity to paraffin oil, } \\
\mathrm{cm}^{3} / 100 \mathrm{~g}\end{array}$ \\
\hline 1 & 20 & 289 & 150 & 250 & 400 \\
\hline 2 & 40 & 256 & 200 & 200 & 450 \\
\hline 3 & 60 & 286 & 250 & 400 & 500 \\
\hline 4 & 80 & 212 & 250 & 400 & 600 \\
\hline
\end{tabular}

\section{RESULTS AND DISCUSSION}

The physicochemical parameters of chromium(III) silicates, precipitated from the system of two emulsions are listed in Table 1.

Particle size distributions of chromium(III) silicates precipitated at various temperatures are presented in Fig. 2 and SEM electron micrograph of sample 2 is shown in Fig. 3.

Chromium(III) silicate obtained at the temperature of $20^{\circ} \mathrm{C}$ (sample 1) manifested in the particle size distribution (Fig. 2a) two bands of different intensities. The more intense band of primary particles and primary agglomer-

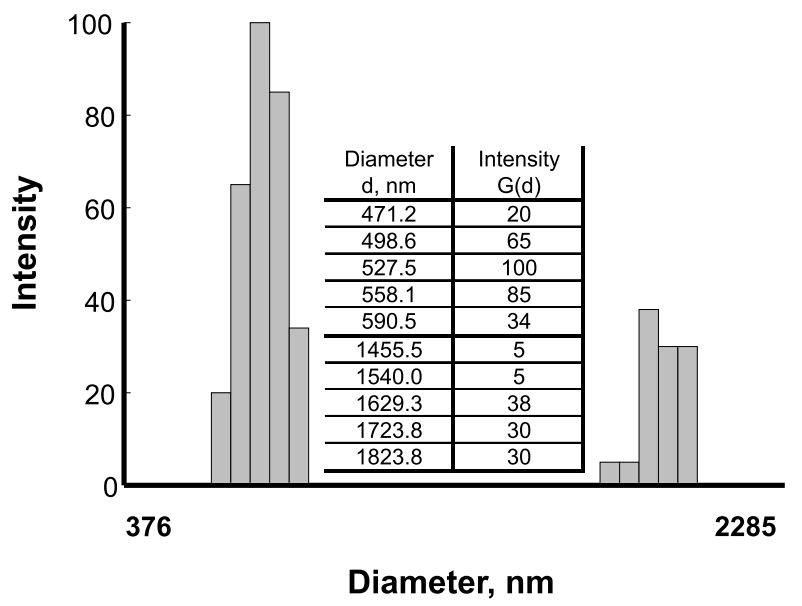

\section{Polydispersity $\mathbf{0} 0.139$ \\ Mean particles diameter $\mathbf{-} 838 \mathrm{~nm}$}

(a) Sample 1

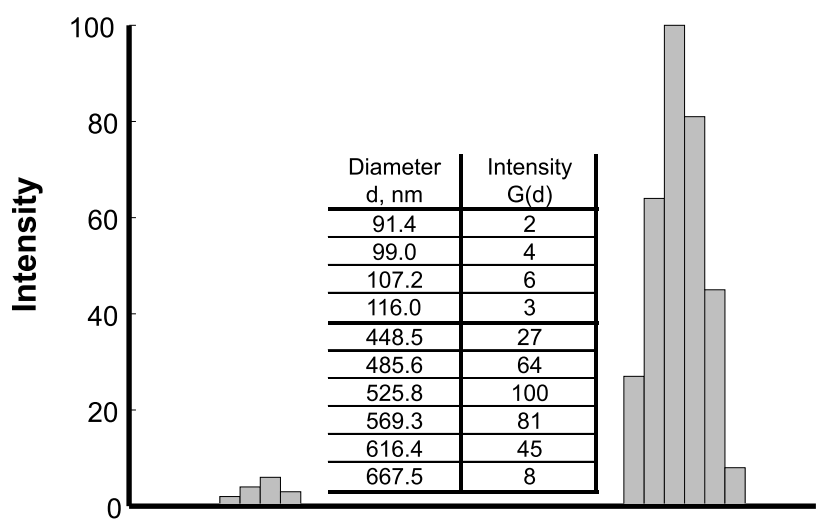

66

Diameter, $\mathbf{n m}$

\section{Polydispersity $\mathbf{- 0 . 2 4 5}$ \\ Mean particles diameter $\mathbf{- 5 1 8} \mathbf{n m}$}

(c) Sample 1 ates fitted the range of $471-590 \mathrm{~nm}$ (maximum intensity of 100 corresponded to the particle of $527.5 \mathrm{~nm}$ in diameter). On the other hand, secondary agglomerates formed a band of lower intensity in the range of $1455-1823 \mathrm{~nm}$ (maximum intensity of 38 corresponded to the agglomerates of $1629.3 \mathrm{~nm}$ in diameter). Sample 2 precipitated at the temperature of $40^{\circ} \mathrm{C}$ demonstrated the presence of two bands in the particle size distribution (Fig. 2b). The more intense band in the range of $357-531 \mathrm{~nm}$ (maximum intensity of 100 corresponded to the particles of $453.7 \mathrm{~nm}$ in diameter) represented primary particles while the faint band of a practically negligible intensity, present at 67 $\mathrm{nm}$, represented very fine primary particles, which was

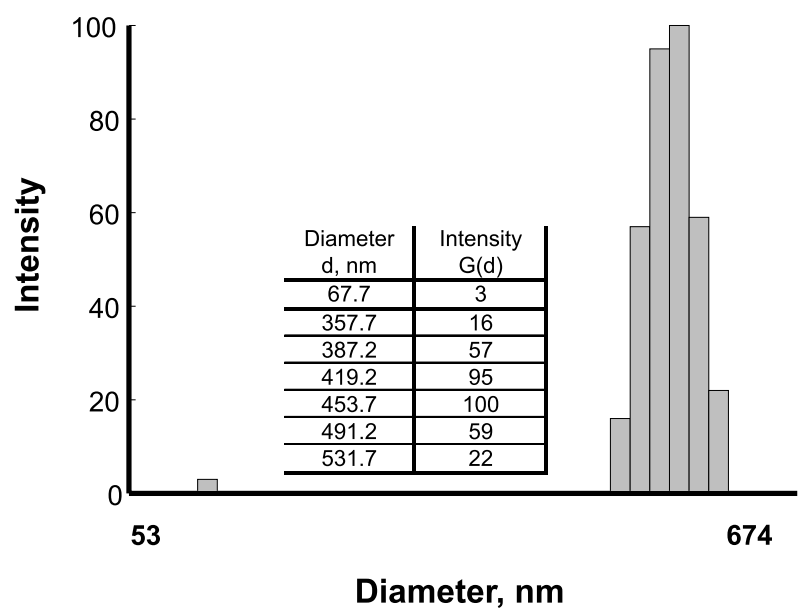

Polydispersity $\mathbf{- 0 . 1 3 5}$ Mean partilces diameter $-437 \mathrm{~nm}$

(b) Sample 2

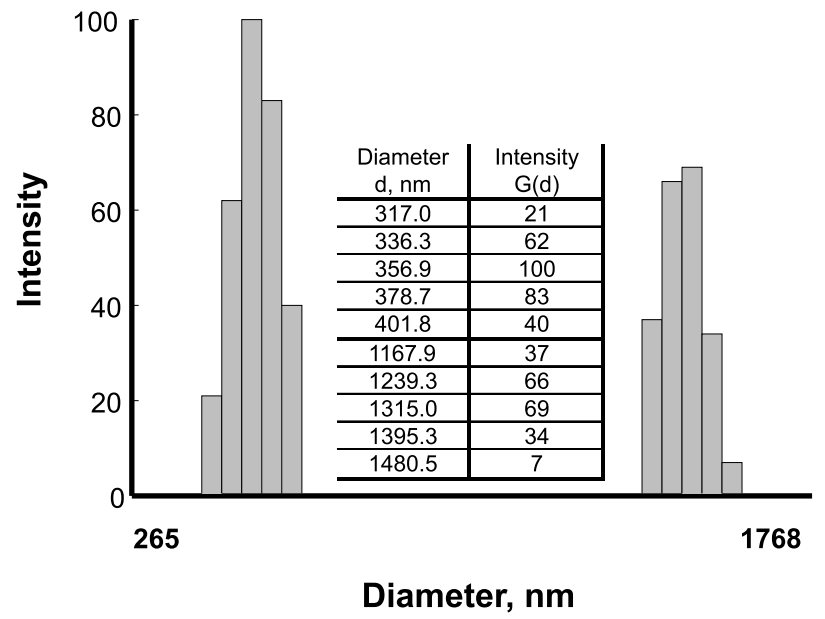

Polydispersity -0.244 Mean particles diameter - $741 \mathrm{~nm}$

(d) Sample 2

Figure 2. Particle size distributions of chromium(III) silicates precipitated at various temperatures: (a) $20^{\circ} \mathrm{C}$; (b) $40^{\circ} \mathrm{C}$; (c) $60^{\circ} \mathrm{C}$ and (d) $80^{\circ} \mathrm{C}$ 


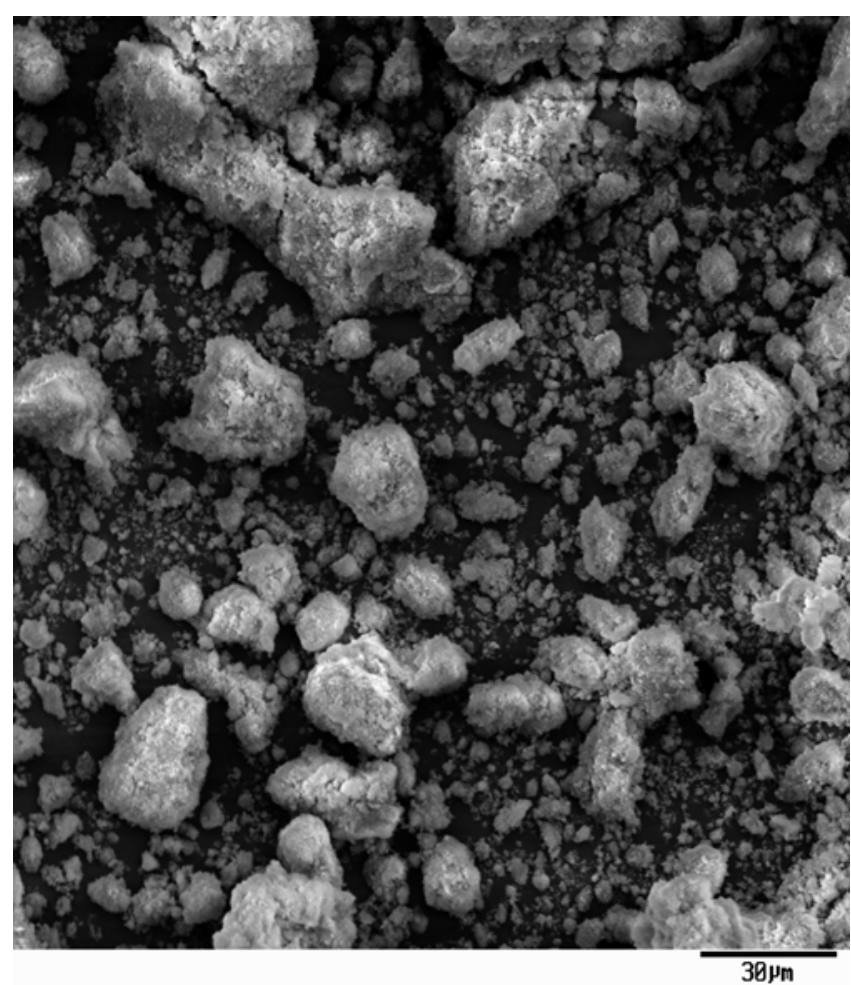

Figure 3. SEM microphotograph of chromium(III) silicate, sample 2

confirmed by the micrograph (Fig. 3). Sample 2 manifested small mean particle diameter of $437 \mathrm{~nm}$ and an average polydispersity -0.135 . A similar situation could be noted in the case of sample 3 (precipitated at the temperature of $60^{\circ} \mathrm{C}$ ) which demonstrated two bands of different intensities (Fig. 2c). The first band represented the accumulation of small primary particles in the range of $91-116 \mathrm{~nm}$ (maximum intensity of 6 corresponded to the particles of $107.2 \mathrm{~nm}$ in diameter). The other band could be ascribed to primary agglomerates (aggregates) in the range of $448-667 \mathrm{~nm}$ (maximum intensity of 100 corresponded to the agglomerates of $525.8 \mathrm{~nm}$ in diameter).

The tendency to form primary particles and secondary agglomerates was demonstrated by sample 4 (Fig. 2d, precipitation temperature of $80^{\circ} \mathrm{C}$ ). The sample contained two bands of different intensities. The more intense band in the diameter range of $317-401 \mathrm{~nm}$ (maximum intensity of 100 corresponded to the particles of $356.9 \mathrm{~nm}$ in diameter) represented primary agglomerates while the band of slightly lower intensity represented secondary agglomerates in the diameter range of $1167-1480 \mathrm{~nm}$ (maximum intensity of 69 corresponded to the agglomerates of $1315 \mathrm{~nm}$ in diameter).

Colour intensities of chromium(III) silicates obtained by precipitation using the system of two emulsions are shown in Fig. 4.

Silicate pigments precipitated from the two emulsion systems manifested green colour, which was most intense in sample 4 , as confirmed by the parameter values of $\mathrm{L}^{*}$ and $\mathrm{a}^{*}\left(\mathrm{~L}^{*}=68, \mathrm{a}^{*}=-16,2\right)$. The remaining pigments, due to their higher value of lucidity $L^{*}$ and lower values of a* index, manifested a slightly less intense green colour.

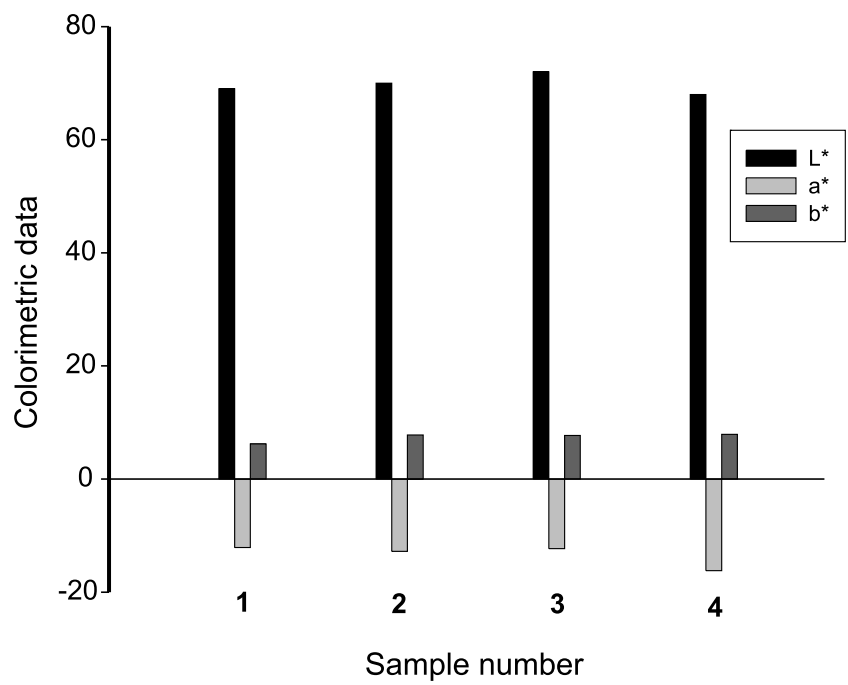

Figure 4. Colour intensity of chromium(III) silicates precipitated in the system of two emulsions at various temperatures

\section{CONCLUSIONS}

The studies performed on the precipitation of green pigments using the novel technique of two emulsions employing cyclohexane as the organic phase and Rokanol K7 as the emulsifier, sodium metasilicate and chromium(III) sulphate solutions as substrates, permitted to obtain highly dispersed pigment particles of extensively uniform character.

The physicochemical properties of the obtained pigments depended on the temperature of precipitation. The studies proved that valuable pigments can be obtained from the waste post-galvanic solutions (originating from chromate coating). However, such use is pre-conditioned by the preliminary reduction of $\mathrm{Cr}^{+6}$ to $\mathrm{Cr}^{+3}$, since only chromium(III) silicates are acceptable for toxicological reasons.

\section{ACKNOWLEDGEMENTS}

This work was supported by the Poznan University of Technology research grant 32-117/07-BW.

\section{LITERATURE CITED}

(1) Krysztafkiewicz A.: Modified calcium silicates as active rubber fillers, J. Mater. Sci., 1987, 22, 478.

(2) Krysztafkiewicz A., Modified zinc silicate - an active rubber activator and filler, J. Mater. Sci., 1988, 23, 2407.

(3) Krysztafkiewicz A., Werner R., Lipska K. L., Jesionowski T.: Effect of silane coupling agents on properties of precipitated sodium-aluminium silicates, Colloids Surf. A, 2001, $182,73$.

(4) Klapiszewska B., Krysztafkiewicz A., Jesionowski T.: Pigment Resin Technol., 2005, 34, 139.

(5) Lambourne R., Strivens T. A.: Pain and Surface Coating, Woodhead Publishing Ltd., Cambridge, 1999.

(6) Endriß H.: Inorganic Coloured Pigments Today, Verlag, Hannover, 1998.

(7) Michalska I., Krysztafkiewicz A., Bogacki M. B., Jesionowski T.: Preparation and characterization of precipitated zinc silicate, J. Chem. Technol. Biot., 2003, 78, 452.

(8) Klapiszewska B., Krysztafkiewicz A., Jesionowski T.: Highly dispersed green silicate and oxide pigments precipitated from post-galvanic refuse, Environ. Sci. Technol., 2003, $37,4811$. 